\title{
Predictive Dialing Call Center Leads to Gain Performance Driven Results in Workflow Management
}

\author{
S. Khaleel Ahamed
}

\begin{abstract}
Today, outbound contact centers are used by businesses for a wide range of purposes- including telemarketing, sales, surveys, fundraising, and other events. While these practices may produce positive business results, contact center agents and managers also need the right tools to manage outbound operations and maximize customer experience although reducing costs. Using the right mix of outbound communications tools can strengthen business outcomes, In particular as the number of contact centers is rising, the transition from cost centers to profit centers. Sixty-nine percent of strategic decision-makers for contact centers in the U.S. and U.K. believe they are critical revenue generators for their organizations, according to Loudhouse Research. Arming agents with the right outbound communication tools is a critical requirement for success, particularly since the bulk of outbound activities are labor-related and companies are looking to generate the greatest possible productivity from their agents.
\end{abstract}

Index Terms - Bigdata, Predictive Dialing, ACD, IVR, Outbound, Inbound.

\section{INTRODUCTION}

Strengthening outbound opportunities With the right outbound communications resources in place, business benefits and operational advantages will make a huge difference. For instance, many organizational leaders are concerned with adhering to industry-specific compliance requirements. This includes agents adhering to explicit communications requirements with customers under regulations such as the Telephone Communications Protection Act (TCPA) which prohibits the use of auto dialers and pre-recorded messages to wireless devices and other numbers. Especially when the call recipient has not given prior consent to be contacted and must pay for the charges.

In these circumstances, preview dialing tools can enable an agent to know who she is going to be connected with, along with pertinent details about that customer in advance.

\section{RELATED WORK}

The right outbound communications tools can also improve the agent experience, which ultimately strengthens the customer experience. For instance, a predictive dialing solution that eliminates the awkward pause that typically occurs when an outbound agent is connected to a customer can enable an agent to immediately engage with a customer.

Revised Manuscript Received on October 25, 2019

Dr.S. Khaleel Ahamed, Associate professor, Department of Computer Science and Engineering, Bharath College of Engineering and Technology for women, Kadapa. E-mail: khaleelska@gmail.com
This preserves the customer experience. The agent doesn't have to over-come any customer against that she's being connected to a telemarketer. The agent can start on a stronger note by having a more natural conversation with the customer. Advanced predictive dialing technologies are just one of the outbound communications tools that companies can leverage to heighten operational and business performance. In the pages that follow, we'll explore the five ways to make the most from outbound communications.

Having the right outbound communication tools in place is critical because it provides companies the ability to execute effectively on outbound campaigns based on their goals. Having the right outbound com-munication tools in place can also help separate the wheat from the chaff. Indeed, nearly two-thirds of best-in-class companies (top 20 percent analyzed) have proactive outbound customer communications processes in place compared to just 28 percent of other companies. We offer five ways for companies to make outbound communications sparkle.

\section{A. Connect outbound gents to customers}

The use of an outbound dialing solution that eliminates the unnecessary pause that can occur between agents and customers that can also intelligently connect the right agent to the right customer via predictive dialing. Creating immediate connections between outbound agents and customers averts hang ups and significantly strengthens business outcomes through improved customer experiences.

An intelligent predictive dialing solution offers other benefits. With most auto dialers, employees have to upload a contact list from a customer database and merge it with the auto dialer, a time-consuming approach where both systems are struggling to manage the customer record. An intelligent predictive dialer can be directly connected to a back-end system such as a CRM system. "So instead of going and getting water by the bucket and loading it one at a time, we're turning on the spigot and making the water available as needed," says Tomczak.

\section{B. Enable intelligent call suppression}

Intelligent call suppression capabilities review the number being queued up for dialing to determine if it is located in the suppression database, allowing agents to handle customer contacts that are most likely to be pro-ductive. Intelligent call suppression can also help agents and supervisors censor leads in real time. This includes the ability to implement Do Not Call restrictions to de-termine whether the contact number is a cell phone or a landline for meeting TCPA requirements; or to prevent an outbound call to a customer who is dialing in. 
Intelligent call suppression can also enable com-panies to strengthen customer loyalty by subverting unnecessary calls that can aggravate customers and damage the company-customer relationship, says Tomczak.

\section{Connect agents to the right data sources}

Cloud-enabled access to CRM and other customer re-cords can arm agents with the right information needed to engage with customers and prospects effectively. This can enable agents to provide customers with timely, relevant service based on what's known about them. For instance, if contact records indicate that a particular customer calls the contact center for a credit card com-pany close to the same date each month to check on their balance and amount that's due, an agent can offer to set up an automated payment plan for that customer that's convenient and less time consuming.

\section{Customize screens and Scripting}

Customized screens and scripting allows agents to focus on the tasks that can drive optimal outcomes (e.g., next best action on collection processes with customers). It is also a way to prompt agents through various levels of questions naturally using a decision tree so it doesn't sound scripted based on the customer's response," says Tomczak.

\section{E. Blend outbound and inbound call traffic}

When inbound call traffic is high and inbound agent resources are strained, outbound agents can be au-tomatically moved to handle inbound calls and then intelligently shifted back to outbound once inbound service level goals have been met. Blending allows contact centers to make the most

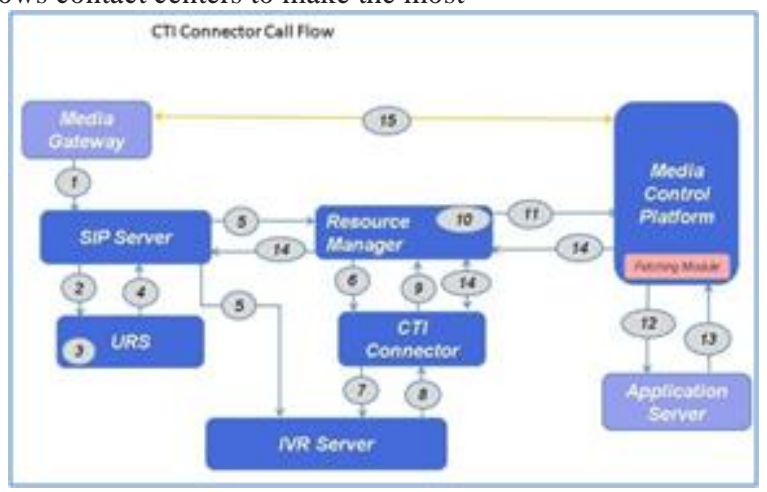

Fig. 1. System Architecture

productive use of subject matter experts (SMEs) for a particular product area or knowledge area or even for a particular customer. In addition, intelligent blending also enables the contact centers to maximize agent performance by filling their day with outbound calls when there is a slowdown on inbound calls or vice versa.

\section{CTI CALL CENTER CONNECTOR WORKFLOW MANAGEMENT}

Figure 1 shows the system design and call routing analysis. Protocol) Server-SIP Header contains ANI and DNIS protocol call data. In this case, the initial destination in Managed Router is a URS-controlled point of access to the route point. SIP is an equipotent peer client-server protocol. In the communication endpoints, SIP technologies are Media Gateway sends Invite to SIP(Session Initiation

introduced, while the standard SS7 architecture is only used in switching centers. Elements of the network. The network components used for interaction using the Session Initiation Protocol are called SIP user agents.

SIP Server provides service providers and enterprises with a SIP-based communication platform. The software has original features of NAT traversal as well as dynamic routing control functions.

As the suggested technique, the call center should set up the SIP trunk network. In the Skype for BusinesslMicrosoft Lync environment, the SIP trunk system enables Enterprise Voice (EV) functionality. There are many benefits to converting the TDM trunks to SIP trunks from cost savings to consolidation of infrastructure. When consolidating the organization's SIP trunks, I have experienced companies save a lot of costs. There are different factors in my personal opinion that contribute to cost savings during the disk. Out of all factors, better SIP consolidation planning contributes the most in cost saving. You can use our SIP Trunk Service to setup either Centralized SIP trunking or SIP trunking distributed. Centralized SIP trunking routes through your central site through all Voice over Internet Protocol (VoIP) traffic, including branch traffic. Thus, SIP trunking routes transmitted VoIP traffic directly from the branch to our SIP gates without going through the central site.

I can use either type of the following connection types for SIP trunking :

Multiprotocol Label Switching(MPLS) that is using a private connection with no other traffic Internet. Configure firewall rules to allow connection with SBCs. Following Table 1 depicts the firewall requirement to make a SIP trunk successfully work.

\begin{tabular}{|l|l|l|l|}
\hline $\begin{array}{l}\text { Gateway IP } \\
\text { Addresses }\end{array}$ & Protocol & Direction & Port \\
\hline x.x.x.x & TCP & $\begin{array}{l}\text { Bi-Direction } \\
\text { al }\end{array}$ & $\begin{array}{l}5060,5061, \\
5067\end{array}$ \\
\hline
\end{tabular}

Table 1. SIP Trunk

You need to decide the protocol (TCP\TLS) to be used for SIP trunk setup. Based, on the selected protocol the specific port would needs to be opened between the mediation server and gateways (of SIP Trunk service providers). You need to add following IP addresses as PSTN gateway in the Skype for Business (SfB) \Microsoft Lync setup.

10.10.10.1 is the gateway IP address of the SIP trunk service provider. You need to be the member of CSAdministrator group to run following steps.

1. Click Start, click All Programs, click Skype for Business Server 2015, and then click Skype for Business Server 2015Topology Builder.

2. Under Skype for Business Server, your site name, Shared Components, right-click the PSTN Gateways node, and then click New PSTN Gateway. 
3. In Define New IP/PSTN Gateway, type the IP address (10.10.10.1) of the peer, and click Next.

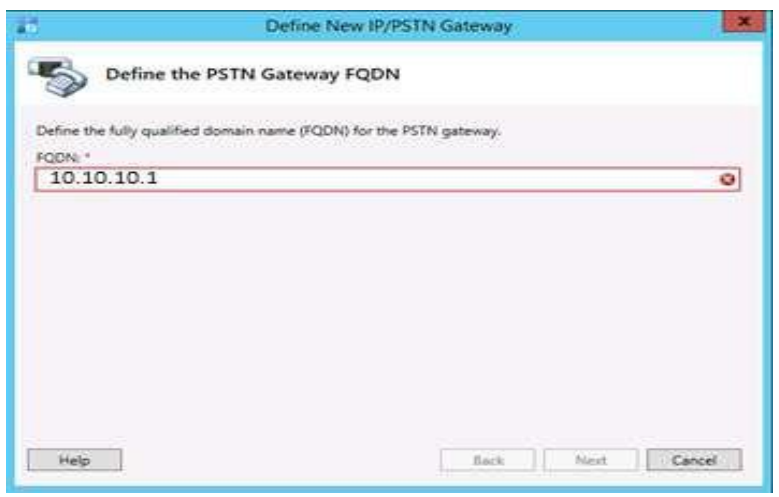

Fig.2. Define the PSTN Gateway FQDN

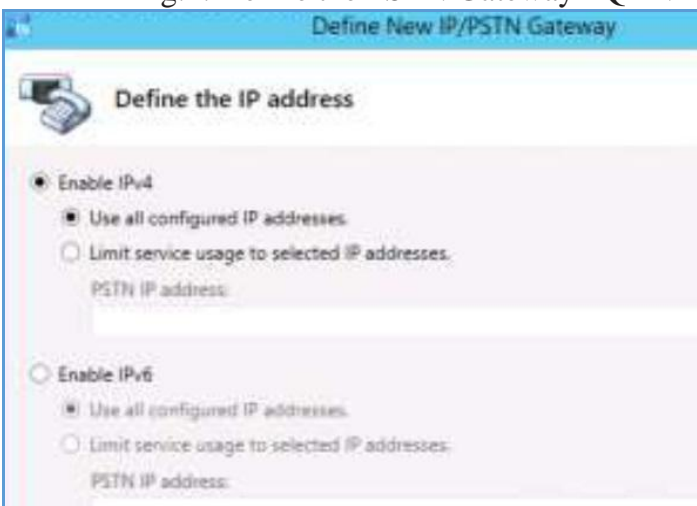

Fig.3 Define New IP/PSTN Gateway

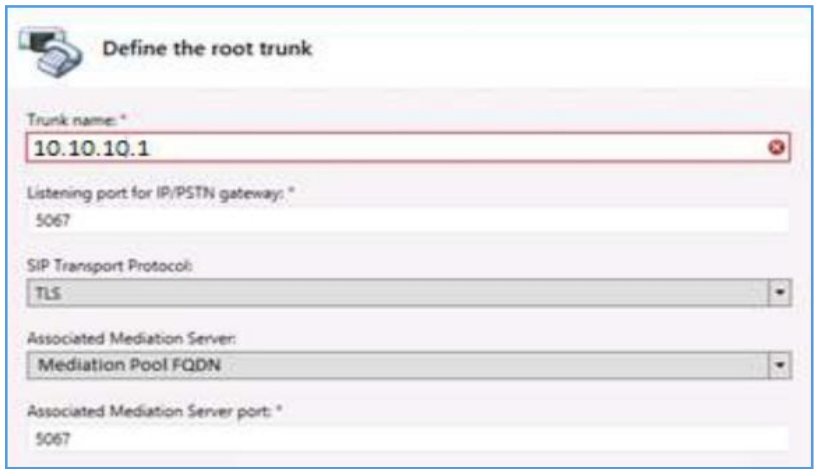

Fig.4. Define the SIP root trunk

Define the port and protocol during above step. You need to get the detail from the SIP trunk service provider. 4.Right-click the Skype for Business Server node, and then click Publish Topology.

\section{F. Configuring Encryption Level (SRTP)}

Encryption support level indicates the level of support for protecting media traffic between the Mediation Server and the PSTN Gateway. Configurable values are:

Required: SRTP encryption must be used.

Optional: SRTP will be used if the gateway supports it.

Not Supported: SRTP encryption is not supported and therefore will not be used.

Select "Optional" as depicted in following diagrams. This needs to be determined with the help of the SIP trunk service provider.

Navigation: Control Panel $=>$ Voice Routing $\Rightarrow>$ Trunk Configuration $=>$ Global $=$ Edit

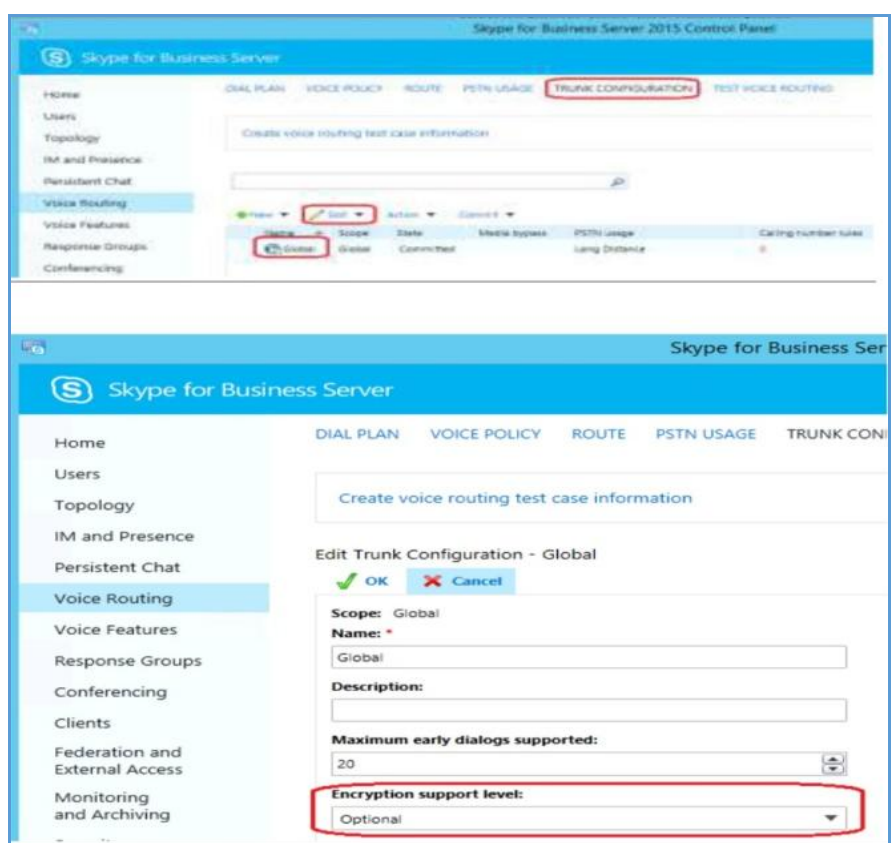

Fig.5. Skype for Business Server in Call Center having optimal encryption support level in SIP trunk setup with configuration details

\section{G. Creating Voice Route}

1. Open Skype for Business Server Control Panel.

2. In the left navigation bar, click Voice Routing.

3. Click Route.

4. Click New to display the New Voice Route dialog box.

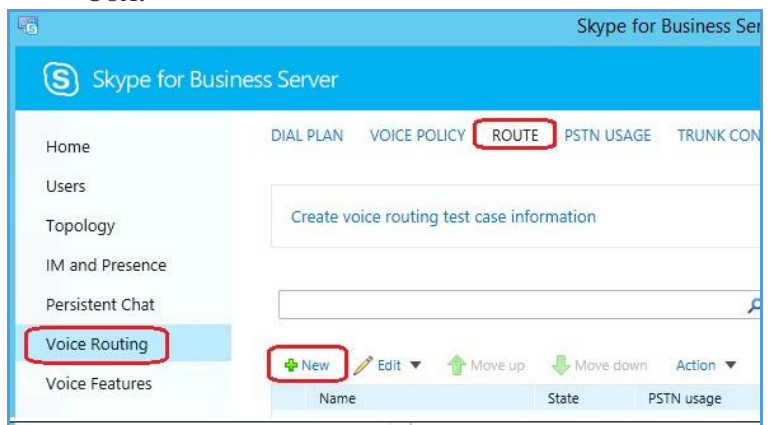

Fig. 6. Voice Routing using the topology i.e. Create voice routing test case information

5. In Name, type a descriptive name for the voice route.

6. To associate one or more trunks with the voice route, click Add and then select a trunk from the list (10.10.10.1).

7. Click OK to save the voice route.

\section{H. Creating Voice Policy}

1. Open Skype for Business Server Control Panel.

2. In the left navigation bar, click Voice Routing.

3. Create a User policy by selecting New. 
Predictive Dialing Call Center Leads to Gain Performance Driven Results in Workflow Management
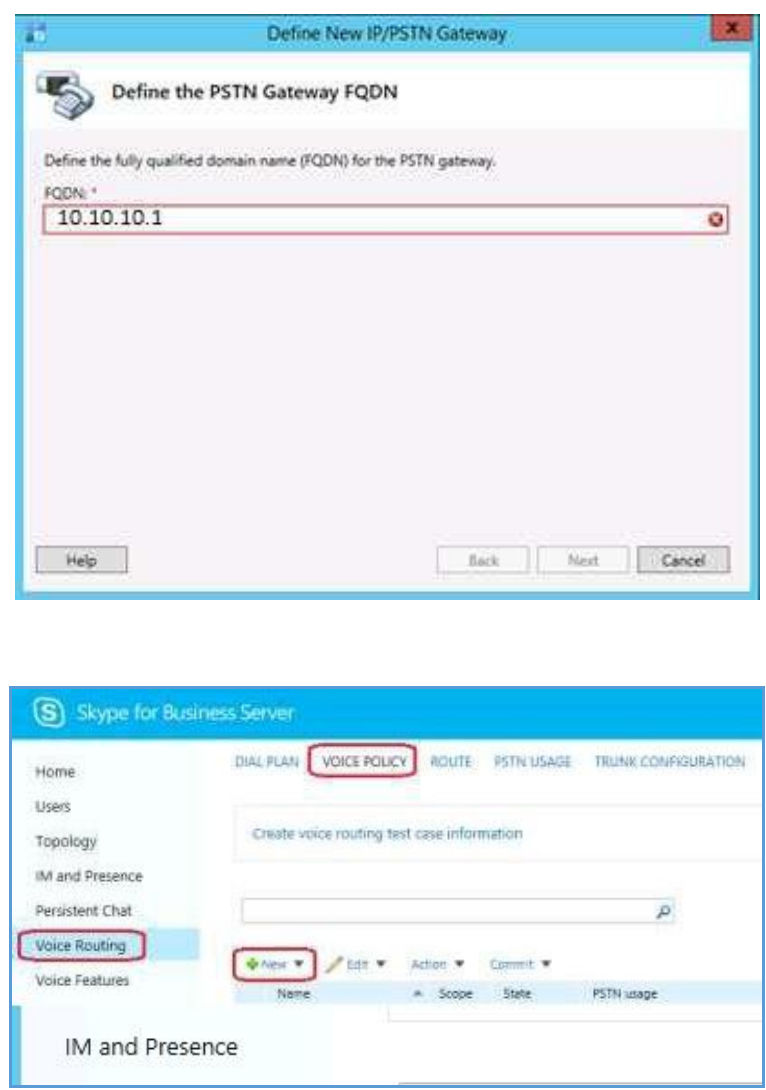

Fig.7. Creating Voice Policy

4. Set Name: Enter a name for this Voice Policy

5. Associate and configuration normalization for the dial plan

6.Verify the dial plan's normalization rules are arranged in the correct order.

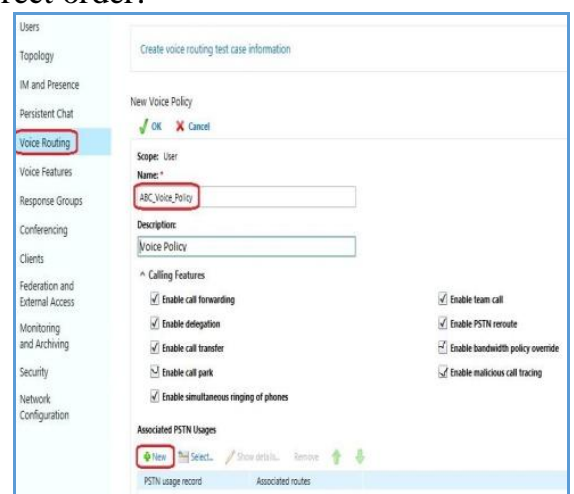

Fig. 8. Voice Routing Mechanism

7.Set Calling Features: In voice routing mechanism, let's check the followings:

1. Enable call forwarding: Checked

2. Enable delegation: Checked

3. Enable call transfer: Checked

4. Enable call park: Unchecked

5. Enable simultaneous ringing of phones: Checked

6. Enable team call: Checked

7. Enable PSTN reroute: Checked

8. Then Click OK On the Dial Plan page, click Commit, and then click Commit all.

9.

\section{Configure Media Bypass}

1. Open Skype for Business Server Control Panel.

2. In the left navigation bar, click Network Configuration and then click Global.

3. Enable 'Enable media bypass' in Global setting.
4. Confirm you have also selected Always bypass

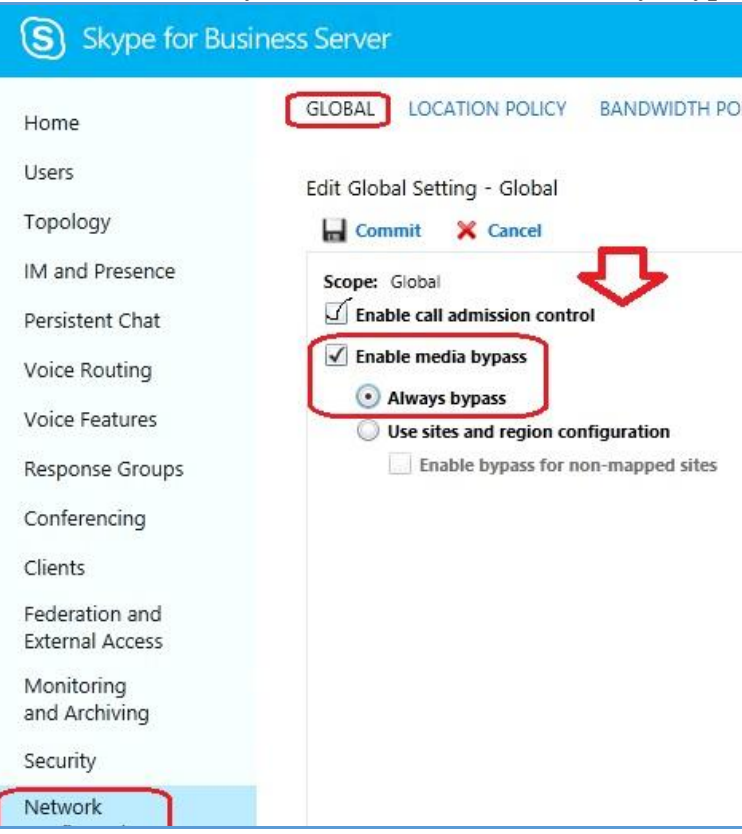

Fig.9 Configuring Media Bypass

\section{J. Enable users for Enterprise Voice (EV)}

1. Enable Skype for Control Panel for Business Server.

2. Click on Users in the left navigation bar.

4. Type all or the first part of the display name, first name, last name, Security Account Manager (SAM) account name, SIP address, or line Uniform Resource Identifier (URI) of the user account you want to allow in the Search users tab, and then click Find.

4. Click on the user account for Enterprise Voice in the list.

5. Tap Show details on the Edit menu.

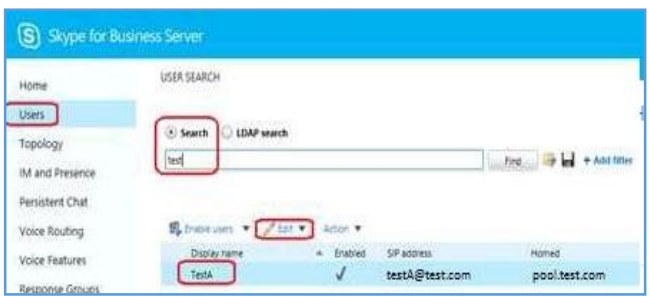

Fig.10. Security Account Manager

6. On the Edit Skype for Business Server User page, under Telephony, click Enterprise Voice.

7. Click Line URI, and then type a unique, normalized phone number (for example, tel:+14255550200).

8. Select Dial Plan and Voice policy.

9. Click Commit.

\section{CONCLUSION}

Testing SIP Trunk through Navigate Control Panel => Voice Routing $=>$ Test Voice Routing $=$ New

Published By:

Blue Eyes Intelligence Engineering 


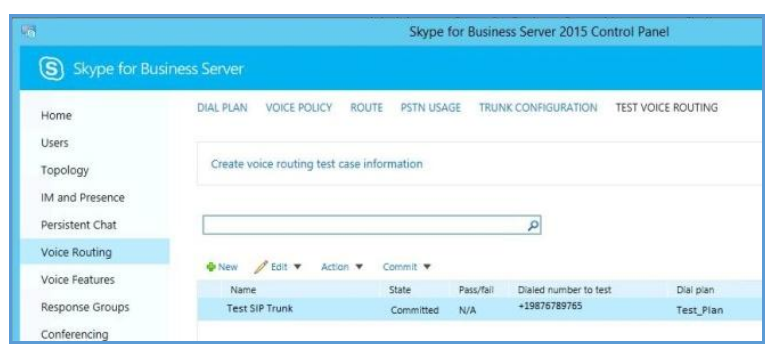

The leadership of the workplace (WFM) sounds more diverse than it is. WFM involves four main processes in the call center environment: predicting the number of customer calls, using the forecasts to create agent schedules that match the service level criteria, assigning such schedules to agents, and ongoing management of changes.

WFM is not an island unconnected to your other activities on its own. A concept you will learn more about in the future is workplace optimization (WFO), which takes a holistic approach to customer relationship management, conducting customer satisfaction surveys, call recording and quality control, training and coaching staff, and providing incentives for success. The ultimate goal, as Gartner's research firm Jim Davies put it in a February 2005 study, is to allow customers to "talk to a professional agent whenever they want, on the platform they want and have a better experience as a result." For instance, Davies suggests that managers could use a performance monitoring tool to determine the training required.

Although we are limiting our discussion in this article to WFM, you should be aware that the practices, and the software that implements them, are growing more interrelated across all of these operational silos.

\section{REFERENCES}

[1]. Aalen, O. O., and Gjessing, H. (2001), "Understanding the Shape of the Hazard Rate: A Process Point of View," Statistical Science, 16, 1-22.

[2]. Anscombe, F. (1948), "The Transformation of Poisson, Binomial and Negative-Binomial Data," Biometrika, 35, 246-254.

[3]. Baccelli, F., and Hebuterne, G. (1981), "On Queues With Impatient Customers," in International Symposium on Computer Performance, ed. E. Gelenbe, Am-sterdam: North Holland, pp. 159-179.

[4]. Benjamini, Y., and Hochberg, Y. (1995), "Controlling the False Discovery Rate: A Pratical and Powerful Approach to Multiple Testing," Journal of the Royal Statistical Society, Ser. B, 57, 289-300.

[5]. Bolotin, V. (1994), "Telephone Circuit Holding Time Distributions," in 14th International Tele-Traffic Conference (ITC-14), Amsterdam: Elsevier, pp. 125-134.

[6]. Borst, S., Mandelbaum, A., and Reiman, M. (2004), "Dimensioning Large Call Centers," Operations Research, 52, 17-34; downloadable at http://iew3. technion.ac.il/serveng/References/references.html.

[7]. Breukelen, G. (1995), "Theorectial Note: Parallel Information Processing Mod-els Compatible With Lognormally Distributed Response Times," Journal of Mathematical Psychology, 39, 396-399.

[8]. Brown, L. D., Gans, N., Mandelbaum, A., Sakov, A., Shen, H., Zeltyn, S., and Zhao, L. (2002a), "Statistical Analysis of a Telephone Call Center: A Queue-ing Science Perspective," technical report, University of Pennsylvania, down-loadable http://iew3.technion.ac.il/serveng/References/references.html.

[9]. Brown, L. D., Mandelbaum, A., Sakov, A., Shen, H., Zeltyn, S., and Zhao, L. (2002b), "Multifactor Poisson and Gamma-Poisson Models for Call Center Arrival Times," technical report, University of Pennsylvania.

[10]. Brown, L. D., and Hwang, J. (1993), "How to Approximate a Histogram by a Normal Density," The American Statistician, 47, 251-255.

[11]. Brown, L., and Shen, H. (2002), "Analysis of Service Times for a Bank Call Center Data," technical report, University of Pennsylvania.
[12]. Brown, L., Zhang, R., and Zhao, L. (2001), "Root Un-Root Methodology for Nonparametric Density Estimation," technical report, University of Pennsyl-vania.

[13]. Brown, L., and Zhao, L. (2002), "A New Test for the Poisson Distribution," Sankhy ${ }^{-}, 64,611-625$.

[14]. Call Center Data (2002), Technion, Israel Institute of Technology, download-able http://iew3.technion.ac.il/serveng/callcenterdata/index.html. 